B.W. Chaffee $1,3 *$, S.A. Gansky ${ }^{1,3}$, J.A. Weintraub ${ }^{1,2}$, J.D.B. Featherstone ${ }^{1,3}$, and F.J. Ramos-Gomez ${ }^{1,4}$

${ }^{1}$ Center to Address Disparities in Children's Oral Health, School of Dentistry, University of California, San Francisco, USA; ${ }^{2}$ School of Dentistry, University of North Carolina, Chapel Hill, USA; ${ }^{3}$ Department of Preventive and Restorative Dental Sciences, School of Dentistry, University of California, San Francisco, USA; and ${ }^{4}$ Section of Pediatrics, School of Dentistry, University of California, Los Angeles, USA; *corresponding author, benjamin.chaffee@ucsf.edu

J Dent Res 93(3):238-244, 2014

\section{Maternal Oral Bacterial Levels Predict Early Childhood Caries Development}

\section{INTRODUCTION}

M ultiple intergenerational connections have been observed between maternal conditions and child health outcomes: from body mass index (Murrin et al., 2012) to age of menarche (Deardorff et al., 2012). Consequently, poor health may be transmitted from parents to children, conceivably perpetuating health disparities and providing reason to consider intergenerational determinants in disease prevention.

Correlations between parental and offspring caries status were recognized at least as early as the 1940s (Klein, 1946). Cross-sectional (Sasahara et al., 1998; Weintraub et al., 2010; Dye et al., 2011) and longitudinal (Grytten et al., 1988; Thitasomakul et al., 2009; Shearer et al., 2012) studies have since corroborated the association between parental dental status and offspring caries, even into adulthood (Shearer et al., 2012). Multiple mechanisms likely underlie intergenerational oral health continuity, including genetic predisposition (Conry et al., 1993), shared social environments (Watt, 2002), and parental oral health knowledge and attitudes (Finlayson et al., 2007), as reviewed by Shearer and Thomson (2010).

Maternal-child transmission of oral bacteria is one such potential mechanism, given the essential, albeit not sufficient, role of oral infection in caries development. Maternal bacterial strains can be detected in children ( $\mathrm{Li}$ and Caufield, 1995), and high maternal salivary mutans streptococci (MS) challenge is associated with earlier child MS acquisition (Li et al., 2005). Mothers of children with caries were more likely to be positive for salivary MS than mothers of caries-free children in a cross-sectional study in New York (Smith et al., 2002). Elsewhere, higher maternal salivary MS levels were associated with child caries and child salivary MS cross-sectionally but not with child caries 2 yr later (Kishi et al., 2009).

The present study follows a birth cohort of low-income Hispanic children whose mothers received repeated dental and salivary bacterial assessments for both MS and lactobacilli (LB) during their children's first $3 \mathrm{yr}$ as part of the Mothers and Youth Access caries prevention trial (Ramos-Gomez et al., 2012). We hypothesized that higher maternal challenge of MS and LB averaged over this period would be associated with greater caries incidence among children.

\section{MATERIAL \& METHODS}

\section{Ethics}

Institutional review boards at collaborating universities (University of California, Los Angeles; University of California, San Francisco; and San 
Diego State University) approved this study. A community advisory board guided study design. Mothers granted informed consent in English or Spanish. Mothers and/or children with caries were referred for treatment.

\section{Participants}

The design and results of the intervention trial in which this prospective observational cohort is nested are presented elsewhere (Ramos-Gomez et al., 2008; Ramos-Gomez et al., 2012). Briefly, the nesting trial randomly assigned 361 mother-infant dyads either to an intervention of oral health counseling, preventive child fluoride varnish applications, and maternal chlorhexidine rinses from 4 to 7 months' postpartum or to a control of oral health counseling and "rescue" (therapeutic) fluoride varnish for children with initial carious lesions. Families were advised to obtain fluoride varnish only through the trial. At 9 months, the intervention reduced maternal salivary MS but not LB; neither was lowered significantly at any other time point (Appendix Table 1). After 36 months, there were no clinically or statistically significant differences in caries between intervention and control group children (Ramos-Gomez et al., 2012).

Participants were registered patients in the second trimester of pregnancy at a health center serving a predominantly lowincome community near the US-Mexico border and without water fluoridation (2003-2007). Trial eligibility criteria included maternal age of 18 to $33 \mathrm{yr}$, singleton fetus, and local residency with evidence of geographic stability (e.g., proof of address). Excluded were sisters or household members of previously enrolled participants, women intending to give birth in Mexico, high-risk pregnancies, and women with existing medical conditions, pregnancy complications, or prior pregnancy-related issues that required hospitalization. Following enrollment, mothers and children returned for questionnaires, dental assessments, and saliva collection at visits corresponding to 4, 9, 12, $18,24,30$, and 36 months' postpartum. The present cohort study includes all mother-child dyads with a 36-month child dental assessment $(N=243)$.

\section{Caries}

Trained, calibrated dental examiners followed standard diagnostic criteria (National Institute of Dental Research, 1991), as described elsewhere (Ramos-Gomez et al., 2012). We calculated the number of decayed (cavitated), missing, or restored permanent teeth (excluding third molars) among mothers (i.e., DMFT index) and the number of decayed (cavitated) or restored primary teeth among children $\left(\mathrm{d}_{2} \mathrm{ft}\right)$. The primary outcome was the cumulative incidence of $\geq 1$ decayed or restored tooth $\left(\mathrm{d}_{2} \mathrm{ft}>0\right)$ at the child's 36-month visit. Mothers were considered to have persistent untreated decay if presenting with $\geq 1$ decayed tooth at all visits ( $97 \%$ completed $\geq 6$ assessments).

\section{Salivary Bacteria}

Bacterial assessments are detailed elsewhere (Ramos-Gomez et al., 2012). Saliva samples $(1.5-2.0 \mathrm{~mL})$ were collected from mothers during pregnancy and with their children at the 4-, 9-, $12-, 24-$, and 36-month visits. Saliva was refrigerated until laboratory transfer for serial dilution and plating within $24 \mathrm{hr}$.
Following 48- to 72-hr anaerobic incubation, colony-forming units per milliliter (CFU/mL) of saliva of MS (Streptococcus mutans and Streptococcus sobrinus) and LB were enumerated.

The primary exposures were the means of the maternal $\log _{10}$ $\mathrm{CFU} / \mathrm{mL}$ levels for MS and for LB, averaged across visits from baseline to 24 months ( $95 \%$ provided $\geq 4$ samples) and dichotomized as higher bacterial challenge (yes/no). Our a priori cutpoint for higher MS challenge ( $4500 \mathrm{CFU} / \mathrm{mL}$ ) was based on a previous study of maternal salivary bacteria and child caries (Kishi et al., 2009). We identified no analogous study for LB; our a priori LB cutpoint $(50 \mathrm{CFU} / \mathrm{mL})$ placed roughly the same proportion of mothers in the higher LB group as in the higher MS group.

\section{Questionnaires}

At each visit, trained bilingual study staff administered maternal questionnaires in English or Spanish, assessing sociodemographic and behavioral factors potentially related to caries. Data were entered into a secure web-based data management system with automated validation checks.

\section{Power}

Recruitment and follow-up in the nesting intervention trial determined the number of participants available for this observational study. We estimated that 188 mother-child dyads would be necessary for $>80 \%$ power to detect a 2 -fold difference in $36-$ month caries incidence $(20 \%$ vs. $40 \%)$ between lower and higher bacterial challenge groups, assuming that $60 \%$ of mothers would be in the higher challenge group (2-tailed alpha $=$ $0.05)$. With 243 eligible dyads, we deemed the statistical power sufficient for multivariable analyses.

\section{Statistical Analysis}

Bivariable comparisons were assessed with 2-tailed hypothesis tests (alpha $=0.05)$ for dichotomous (chi-square), normally distributed continuous (Student $t$ test), or nonnormally distributed continuous (Mann-Whitney $U$ test) variables. General linear mixed effects models (clustering by mother-child dyads; unstructured correlation) were used to compare maternal salivary bacteria levels $\left(\log _{10} \mathrm{CFU} / \mathrm{mL}\right)$ over time by child caries status, separately for MS and LB.

In the primary analysis, the parameter of interest was the cumulative incidence ratio (relative risk) of caries $\left(\mathrm{d}_{2} \mathrm{ft}>0\right)$ comparing children of mothers with higher bacterial challenge to those with lower challenge. Separately for MS and LB exposures, unadjusted and adjusted log-linear regression models were estimated. Covariates were selected a priori considering possible causal roles in the caries process. The 3 nested adjusted models progressively added categories of variables. First, sociodemographic variables were included: maternal education ( $<$ high school or equivalent $v s$. $\geq$ high school), non-English language exclusively at home (yes/no), family structure (both parents live in household, yes/no), annual household income $(<\$ 7000, \$ 7000-\$ 35,000,>\$ 35,000)$, nationality (Mexican or Mexican American $v s$. other), and parity (child is first born, yes/ no). Second, feeding and care behaviors were added: breastfeeding duration (0-2, 3-9, or $\geq 10$ months), daily child tooth-brushing assistance with fluoride toothpaste at 24 months (yes/no), utensil 
Table 1. Descriptive Characteristics and Dental Health of Study Participants: Eligible Mother-Child Dyads $(N=243)^{\mathrm{a}}$

\begin{tabular}{|c|c|c|}
\hline Characteristic & $n(\%)$ & $M \pm S D$ (Range) \\
\hline \multicolumn{3}{|l|}{ Maternal and household sociodemographics } \\
\hline \multicolumn{3}{|l|}{ Mother identifies as } \\
\hline Mexican and/or Mexican American & $230(95.4)$ & \\
\hline Non-English language used exclusively at home & $154(63.9)$ & \\
\hline Mother less than high school degree or equivalent & $101(42.3)$ & \\
\hline$<7000$ & $52(22.2)$ & \\
\hline 7000 to 35,000 & $159(67.9)$ & \\
\hline$\geq 35,000$ & $23(9.8)$ & \\
\hline Child is mother's first & $103(42.4)$ & \\
\hline Both parents and child live in same household & $164(67.8)$ & \\
\hline \multicolumn{3}{|l|}{ Child feeding and care practices } \\
\hline Brushing with fluoride toothpaste and caregiver help at 24-month visit & $107(45.9)$ & \\
\hline Mother ever shared utensils with child & $55(23.0)$ & \\
\hline \multicolumn{3}{|l|}{ Total daily frequency of meals and snacks at 24 months } \\
\hline$<8$ & $51(21.9)$ & \\
\hline $8-10$ & $151(64.8)$ & \\
\hline$\geq 11$ & $31(13.3)$ & \\
\hline Mother is primary caregiver & $222(92.1)$ & \\
\hline Child ever attended daycare & $71(29.2)$ & \\
\hline \multicolumn{3}{|l|}{ Maternal oral health status } \\
\hline DMFT at baseline & & $12.7 \pm 5.0(1-26)$ \\
\hline DT at baseline & & $7.3 \pm 5.3(0-22)$ \\
\hline Children with untreated decay $\left(d_{2} \dagger>0\right)$ & 75 (30.9) & \\
\hline $\mathrm{d}_{2} \mathrm{ft}$ & & $1.41 \pm 2.54(0-12)$ \\
\hline Child $\log _{10} M S$ & & $2.5 \pm 2.5(0-7.5)$ \\
\hline Children MS positive & $121(55.3)$ & \\
\hline Child $\log _{10} L B$ & & $0.5 \pm 1.1(0-5.6)$ \\
\hline Children LB positive & $39(16.4)$ & \\
\hline
\end{tabular}

Abbreviations: $d_{2} f t$, decayed (cavitated) filled primary tooth index; $d_{2} t$, decayed (cavitated) primary tooth index; DMFT, decayed, missing, and filled permanent tooth index; DT, decayed permanent tooth index; LB, lactobacilli; MS, mutans streptococci.

a Sample size ranges from 234 to 243 because of missing baseline information for some variables.

bEquivalent to 4500 colony-forming units per milliliter of saliva.

'Equivalent to 50 colony-forming units per milliliter of saliva.

sharing (ever/never), combined daily meal and snack frequency at 24 months $(4-7,8-10, \geq 11)$, mother as primary caregiver (yes/ no), and daycare attendance (ever/never). Last, maternal dental factors were added: baseline decayed teeth (count) and persistent untreated decay (yes/no).

To test whether allocation status in the nesting intervention trial influenced results, models were estimated both restricted to the trial control group $(n=120)$ and pooled across trial groups $(N=243)$, the latter with terms for trial group and Trial Group $\times$ Bacterial Challenge interaction. Restricted and pooled estimates were qualitatively similar, and no statistically significant interactions were detected (all $p>.4$ ). Therefore, the primary results are from pooled models that include a trial group term, even in unadjusted models. For reference, Appendix Table 2 provides restricted results. Secondarily, to evaluate the association with child caries of maternal MS and LB both independently and combined, we estimated a fully adjusted model with terms for high MS, high LB, and MS $\times$ LB interaction. 
Missing covariate data ( $1 \%$ of all data) were addressed with multiple imputation. Point estimates were averaged over 50 imputations. We used nonparametric bootstrap resampling (5000 repetitions) to estimate $95 \%$ confidence intervals that accounted for both sampling variability and imputation. As a sensitivity check, we ran analogous zero-inflated Poisson regression models for the outcome child $\mathrm{d}_{2} \mathrm{ft}$ (Appendix Table 3). Analyses were completed with Stata 12.1 (StataCorp LP) and R 3.0.1 (http://r-project.org). Study reporting followed STROBE guidelines (von Elm et al., 2007).

\section{RESULTS}

Eligible study participants $(N=243$ mother-child dyads) were primarily from low-income Mexican American families (Table 1). Among mothers, caries experience was universal (100\% baseline DMFT $>0)$, and persistent active caries was prevalent ( $58 \%$ with untreated decay at all visits). There was no statistically significant association of any Table 1 variable with treatment allocation in the nesting trial or with postenrollment loss to follow-up. At 36 months, 34\% of children were caries positive $\left(\mathrm{d}_{2} \mathrm{ft}>0\right)$, including $31 \%$ with untreated decay (Table 1), while $53 \%$ and $16 \%$ of children were positive for MS and LB, respectively.

For both MS and LB, maternal salivary bacterial levels - at each visit and averaged over the study period-were higher among mothers of children with caries $\left(\mathrm{d}_{2} \mathrm{ft}>0\right)$ at 36 months versus those caries-free (Figure 1). Compared with children of mothers with lower levels, children of mothers with higher maternal salivary MS averaged from baseline to 24 months were more likely to be MS positive at 36 months (63\% vs. $43 \%, p<.01$ ), although mean $\log _{10}$ MS counts among MS-positive children were similar with or without higher maternal MS challenge (4.6 vs. 4.4, respectively, $p=.42$ ). Higher average maternal LB was not statistically significantly associated with child LB positivity $(19 \%$ vs. $13 \%, p=.18)$ or with mean $\log _{10}$ LB counts among LB-positive children (2.8 vs. 2.8, $p=.94)$.

Higher average maternal salivary bacterial challenge was associated with greater child caries incidence for MS, but this relationship was weaker and not statistically significant for LB
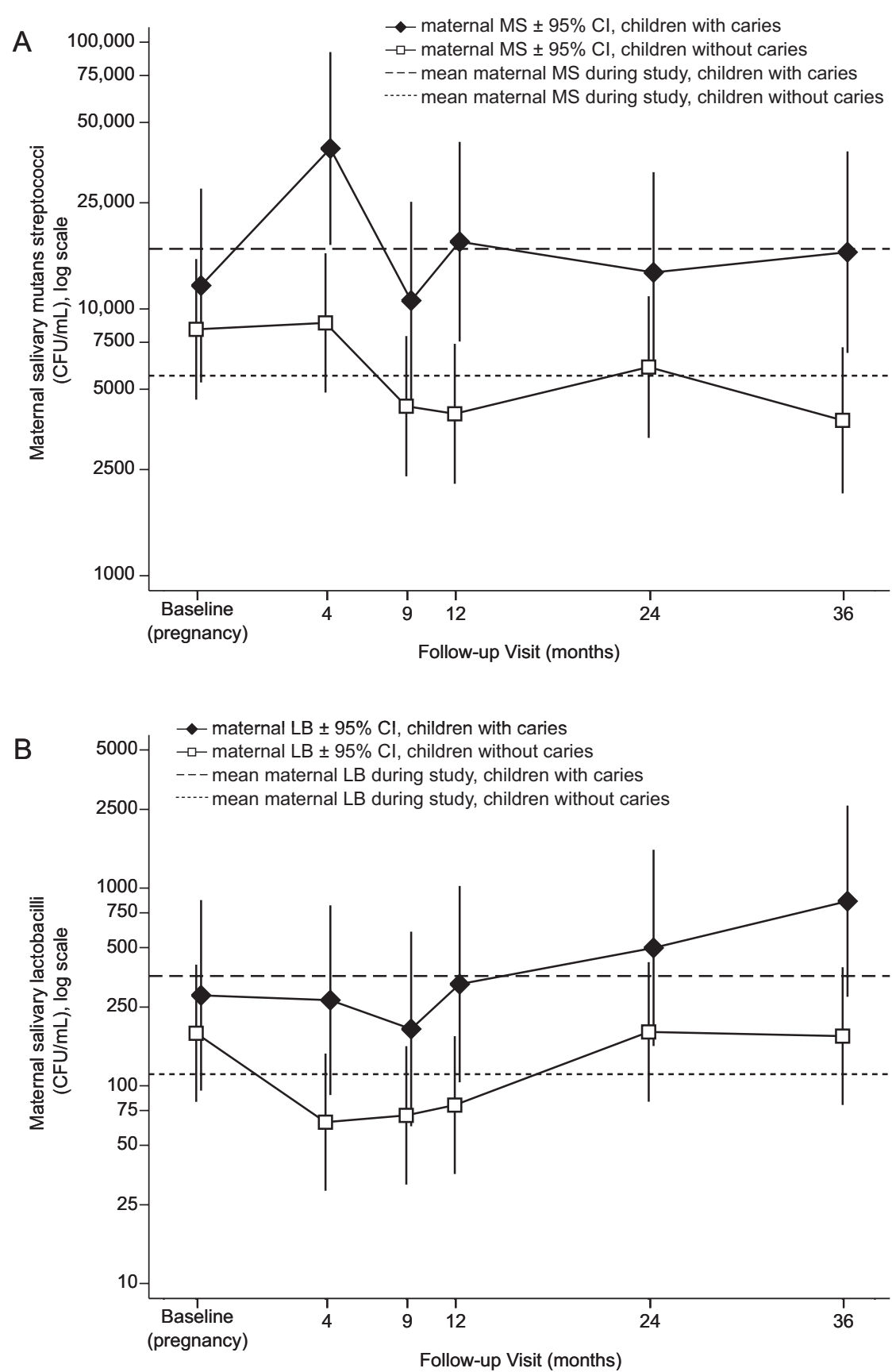

Figure 1. Maternal salivary bacterial counts by child dental status. Maternal salivary bacterial counts of (A) MS and (B) LB by 36-month child caries status $(\geq 1$ cavitated or restored teeth vs. caries-free). Maternal bacterial levels of MS $(p<.01)$ and LB $(p=.03)$ over the study period were greater among mothers of children with caries (Wald test), based on 1369 (MS) and 1405 (LB) salivary measurements clustered in 243 mother-child dyads. Abbreviations: CFU, colony-forming units; $\mathrm{Cl}$, confidence interval; $\mathrm{LB}$, lactobacilli; $\mathrm{MS}$, mutans streptococci.

(Table 2). In the interaction model, high maternal challenge of MS was more strongly associated with greater child caries incidence if maternal LB challenge was low (Table 3). The relative increase in caries prevalence associated with high maternal challenge from MS and LB together (cumulative incidence ratio, $1.9 ; 95 \%$ confidence interval, $1.1,3.8$ ) was not markedly 
Table 2. Child Caries Incidence $\left(d_{2} f t>0\right)$ at 36 Months by Higher versus Lower Mean Maternal Salivary Bacterial Challenge ( $N=243$ )

\begin{tabular}{|c|c|c|c|c|}
\hline Model & Cumulative Incidence Ratio & $95 \%$ Confidence Interval & Cumulative Incidence Ratio & $95 \%$ Confidence Interva \\
\hline Adjusted model $1^{c}$ & 1.7 & $1.1,2.6$ & 1.2 & $0.8,1.8$ \\
\hline Adjusted model $2^{d}$ & 1.8 & $1.2,2.9$ & 1.3 & $0.9,1.9$ \\
\hline Adjusted model $3^{e}$ & 1.7 & $1.1,2.8$ & 1.2 & $0.8,1.8$ \\
\hline
\end{tabular}

Abbreviations: $C F U / m L$, colony-forming units per milliliter saliva; $d_{2} f t$, decayed (cavitated) filled primary tooth index; LB, lactobacilli; MS, mutans streptococci.

'Mean maternal bacterial levels from baseline (in pregnancy) to 24 months' postpartum.

bIncludes allocation status from nesting intervention study only.

Includes allocation status from nesting intervention study and maternal education, language spoken at home, family structure, household income, maternal nationality, and parity.

${ }^{d}$ Includes all adjusted model 1 variables and breastfeeding duration, child tooth brushing, utensil sharing, feeding frequency, mother as primary caregiver, and daycare attendance.

encludes all adjusted model 2 variables and maternal baseline decayed tooth index and persistent untreated maternal decay.

Table 3. Interaction of Maternal Salivary Mutans Streptococci and Lactobacilli Challenge in Association with Child Caries Incidence $\left(\mathrm{d}_{2} \mathrm{ft}>0\right)$ at 36 Months

\begin{tabular}{lllcc}
\hline & Exposure & Reference & $\begin{array}{c}\text { Cumulative } \\
\text { Incidence } \\
\text { Ratio }\end{array}$ & $\begin{array}{c}95 \% \\
\text { Confidence } \\
\text { Interval }\end{array}$ \\
\hline$M S$ & $\begin{array}{l}\text { High } \\
\text { Low }\end{array}$ & Low & 2.4 & $1.3,4.9$ \\
LB & Low & & \\
$n$ & High & Low & 1.2 & $0.6,2.7$ \\
$M S$ & High & High & & \\
LB & 106 & 26 & & \\
$n$ & Low & Low & 1.6 & $0.7,3.7$ \\
$M S$ & High & Low & & \\
LB & 26 & 70 & & \\
$n$ & High & High & 0.8 & $0.5,1.3$ \\
$M S$ & High & Low & & \\
LB & 106 & 41 & & \\
$n$ & High & Low & 1.9 & $1.1,3.7$ \\
$M S$ & High & Low & & \\
LB & 106 & 70 & & \\
$n$ & Ho & & &
\end{tabular}

Abbreviations: $d_{2} f t$, decayed (cavitated) filled primary tooth index; LB, lactobacilli; MS, mutans streptococci; $n$, mothers with this combination of MS/LB challenge.

${ }^{a}$ Cutpoint for higher versus lower maternal mean salivary MS $=4500$ colony-forming units per milliliter of saliva, averaged from baseline (in pregnancy) to 24 months' postpartum.

${ }^{b}$ Cutpoint for higher versus lower maternal mean salivary $L B=50$ colony-forming units per milliliter of saliva, averaged from baseline (in pregnancy) to 24 months' postpartum.

Test for statistical MS $\times$ LB interaction: $p=.14$.

Adjusted for allocation status from nesting intervention study and maternal education, language spoken at home, family structure, household income, maternal nationality, parity, breastfeeding duration, child tooth brushing, utensil sharing, feeding frequency, mother as primary caregiver, daycare attendance, maternal baseline decayed tooth index, and persistent untreated maternal decay.

stronger than that associated with high challenge from either MS alone, suggesting no synergistic relationship.

\section{DISCUSSION}

Poor maternal dental health might enhance caries development in subsequent generations through multiple mechanismsgenetic, behavioral, infectious, and social (Shearer and Thomson, 2010). Here, we demonstrated that, when adjusted for numerous factors, higher maternal salivary bacterial challenge was prospectively, longitudinally associated with child caries in a lowincome Hispanic cohort. Multiple studies have documented maternal-to-child transfer of caries-causing oral bacteria ( $\mathrm{Li}$ and Caufield, 1995; Klein et al., 2004). To our knowledge, this study is the largest prospective longitudinal investigation showing that maternal MS and LB predicted not only oral infection among children but also a meaningful increase in caries incidence. Maternal bacterial challenge continues to merit consideration among the multiple individual, familial, and community risk factors for early childhood caries.

Association estimates changed little after adjustment for various sociodemographic, feeding/care, and maternal dental measures, suggesting limited confounding by these variables. We hypothesize, however, that the association between maternal cariogenic salivary bacteria and child caries might be strongest under contexts entailing close maternal contacts (e.g., breastfeeding, utensil sharing) and weaker under contexts that imply broader potential sources of infection (e.g., daycare attendance, having siblings). For example, greater fidelity of maternal and child MS genotypes was observed among MS-positive children who were breastfed (Li et al., 2000). If observed, such effect measure modification would strengthen the mechanistic evidence inculpating maternal bacterial transfer as a caries determinant among children, representing an area for future study.

While maternal salivary MS and LB levels over the study period were associated with child caries, the nesting intervention trial — which lowered maternal bacterial levels only temporarily and only among a subset of mothers - had no caries effect among children (Ramos-Gomez et al., 2012). Thus, sustained reductions in maternal oral bacterial levels might be more critical than short-term declines for preventing child caries. As a 
methodological consideration, averaging maternal bacterial levels over repeated measurements likely permitted more valid classification of mothers into higher challenge groups in light of intrasubject variability.

This study builds on previous work by considering maternal salivary MS and LB, rather than MS alone, in relation to child caries. However, we did not observe synergistic interaction between MS and LB. Instead, we found that higher maternal challenge from MS or LB alone predicted child caries, albeit not statistically significant for LB, and that each exposure added little information if the other was already present. This contrasts a recent finding in adults, which reported the greatest number of decayed surfaces associated with high levels of MS and LB together (Featherstone et al., 2012). However, because of correlation between MS and LB, fewer mothers were discordant with regard to their salivary challenge from each bacterial group, making the evaluation of statistical interaction more challenging. Thus, our findings await confirmation in larger samples. Several bacteria have been associated with early childhood caries (Tanner et al., 2011). Future studies are needed measuring multiple groups of maternal oral bacteria, individually and in relation to one another.

As a limitation, we did not isolate bacterial strains for genetic analysis and therefore could not directly confirm maternal-tochild transfer. Studies have shown that oral bacteria in children has both maternal and nonmaternal origins (Mitchell et al., 2009; Zhan et al., 2012). As children age, additional sources of oral bacteria (e.g., other caregivers, siblings, peers) are likely to contribute to caries susceptibility. Thus, despite adjustment for numerous factors, unmeasured correlations in genetics, diets, and environments, rather than infection alone, could partially account for interrelationships between maternal and child bacteria. Also, this study population featured a heightened maternal caries burden; whether the results apply more generally requires further investigation. To prevent unnecessary exposure, radiographic examinations were not conducted, potentially lowering sensitivity to detect some carious lesions.

Our study highlights the contribution of maternal bacterial challenge in early childhood caries, supporting ongoing interruption of vertical transmission as a potential preventive strategy. A multifaceted intervention to reduce oral bacterial challenge in mothers was associated with both short- and longterm reductions in MS colonization (Köhler et al., 1984; Köhler and Andréen, 2012) and caries prevention in primary and permanent teeth (Köhler and Andréen, 1994, 2012). Interventions that provided mothers with xylitol-containing chewing gum successfully prevented child MS colonization and reduced child caries (Thorild et al., 2006; Söderling, 2009), although longer duration and higher dose regimens yielded greater effects (Söderling, 2009). Thus, early initiated and sustained interventions, perhaps integrating life course and socioenvironmental determinants perspectives (Kenney et al., 2012), might be best positioned for favorable outcomes. Broadly implemented maternalchild health initiatives might contribute meaningful reductions in oral health disparities by helping to break an intergenerational chain of oral health disadvantage.

\section{ACKNOWLEDGMENTS}

This research made possible by grants from the National Institutes of Health and National Institute of Dental and Craniofacial Research (U54DE014251 and U54DE019285). The authors thank the study participants, the San Ysidro Health Center for its collaboration, and Dr. Charles Hoover of the University of California, San Francisco, for assistance with bacterial assays. The authors declare no potential conflicts of interest with respect to the authorship and/or publication of this article.

\section{REFERENCES}

Conry JP, Messer LB, Boraas JC, Aeppli DP, Bouchard TJ Jr (1993). Dental caries and treatment characteristics in human twins reared apart. Arch Oral Biol 38:937-943.

Deardorff J, Berry-Millett R, Rehkopf D, Luecke E, Lahiff M, Abrams B (2012). Maternal pre-pregnancy BMI, gestational weight gain, and age at menarche in daughters. Matern Child Health J 17:1391-1398.

Dye BA, Vargas CM, Lee JJ, Magder L, Tinanoff N (2011). Assessing the relationship between children's oral health status and that of their mothers. J Am Dent Assoc 142:173-183.

Featherstone JD, White JM, Hoover CI, Rapozo-Hilo M, Weintraub JA, Wilson RS, et al. (2012). A randomized clinical trial of anticaries therapies targeted according to risk assessment (caries management by risk assessment). Caries Res 46:118-129.

Finlayson TL, Siefert K, Ismail AI, Sohn W (2007). Psychosocial factors and early childhood caries among low-income African-American children in Detroit. Community Dent Oral Epidemiol 35:439-448.

Grytten J, Rossow I, Holst D, Steele L (1988). Longitudinal study of dental health behaviors and other caries predictors in early childhood. Community Dent Oral Epidemiol 16:356-359.

Kenney MK, Kogan MD, Toomer S, van Dyck PC (2012). Federal expenditures on maternal and child health in the United States. Matern Child Health $J$ 16:271-287.

Kishi M, Abe A, Kishi K, Ohara-Nemoto Y, Kimura S, Yonemitsu M (2009). Relationship of quantitative salivary levels of Streptococcus mutans and $\mathrm{S}$. sobrinus in mothers to caries status and colonization of mutans streptococci in plaque in their 2.5-year-old children. Community Dent Oral Epidemiol 37:241-249.

Klein H (1946). The family and dental disease; dental disease (DMF) experience in parents and offspring. $J$ Am Dent Assoc 33:735-743.

Klein MI, Florio FM, Pereira AC, Hofling JF, Goncalves RB (2004). Longitudinal study of transmission, diversity, and stability of Streptococcus mutans and Streptococcus sobrinus genotypes in Brazilian nursery children. J Clin Microbiol 42:4620-4626.

Köhler B, Andréen I (1994). Influence of caries-preventive measures in mothers on cariogenic bacteria and caries experience in their children. Arch Oral Biol 39:907-911.

Köhler B, Andréen I (2012). Mutans streptococci and caries prevalence in children after early maternal caries prevention: a follow-up at 19 years of age. Caries Res 46:474-480.

Köhler B, Andréen I, Jonsson B (1984). The effect of caries-preventive measures in mothers on dental caries and the oral presence of the bacteria Streptococcus mutans and lactobacilli in their children. Arch Oral Biol 29:879-883.

Li Y, Caufield PW (1995). The fidelity of initial acquisition of mutans streptococci by infants from their mothers. J Dent Res 74:681-685.

Li Y, Wang W, Caufield PW (2000). The fidelity of mutans streptococci transmission and caries status correlate with breast-feeding experience among Chinese families. Caries Res 34:123-132.

Li Y, Caufield PW, Dasanayake AP, Wiener HW, Vermund SH (2005). Mode of delivery and other maternal factors influence the acquisition of Streptococcus mutans in infants. J Dent Res 84:806-811. 
Mitchell SC, Ruby JD, Moser S, Momeni S, Smith A, Osgood R, et al. (2009). Maternal transmission of mutans streptococci in severe-early childhood caries. Pediatr Dent 31:193-201.

Murrin CM, Kelly GE, Tremblay RE, Kelleher CC (2012). Body mass index and height over three generations: evidence from the Lifeways crossgenerational cohort study. BMC Public Health 12:81.

National Institute of Dental Research (1991). Oral health surveys of the National Institute of Dental Research, diagnostic criteria and procedures. NIH Publication No. 91-2870. Bethesda, MD: NIH-NIDCR.

Ramos-Gomez F, Chung LH, Gonzalez Beristain R, Santo W, Jue B, Weintraub J, et al. (2008). Recruiting and retaining pregnant women from a community health center at the US-Mexico border for the Mothers and Youth Access clinical trial. Clin Trials 5:336-346.

Ramos-Gomez FJ, Gansky SA, Featherstone JD, Jue B, Gonzalez-Beristain $\mathrm{R}$, Santo W, et al. (2012). Mother and Youth Access (MAYA) maternal chlorhexidine, counselling and paediatric fluoride varnish randomized clinical trial to prevent early childhood caries. Int J Paediatr Dent 22:169-179.

Sasahara H, Kawamura M, Kawabata K, Iwamoto Y (1998). Relationship between mothers' gingival condition and caries experience of their 3-year-old children. Int J Paediatr Dent 8:261-267.

Shearer DM, Thomson WM (2010). Intergenerational continuity in oral health: a review. Community Dent Oral Epidemiol 38:479-486.

Shearer DM, Thomson WM, Caspi A, Moffitt TE, Broadbent JM, Poulton R (2012). Family history and oral health: findings from the Dunedin Study. Community Dent Oral Epidemiol 40:105-115.
Smith RE, Badner VM, Morse DE, Freeman K (2002). Maternal risk indicators for childhood caries in an inner city population. Community Dent Oral Epidemiol 30:176-181.

Söderling EM (2009) Xylitol, mutans streptococci, and dental plaque. Adv Dent Res 21:74-78.

Tanner AC, Kent RL Jr, Holgerson PL, Hughes CV, Loo CY, Kanasi E, et al. (2011). Microbiota of severe early childhood caries before and after therapy. J Dent Res 90:1298-1305.

Thitasomakul S, Piwat S, Thearmontree A, Chankanka O, Pithpornchaiyakul W, Madyusoh S (2009). Risks for early childhood caries analyzed by negative binomial models. $J$ Dent Res 88:137-141.

Thorild I, Lindau B, Twetman S (2006). Caries in 4-year-old children after maternal chewing of gums containing combinations of xylitol, sorbitol, chlorhexidine and fluoride. Eur Arch Paediatr Dent 7:241-245.

von Elm E, Altman DG, Egger M, Pocock SJ, Gøtzsche PC, Vandenbroucke JP, et al. (2007). The Strengthening the Reporting of Observational Studies in Epidemiology (STROBE) statement: guidelines for reporting observational studies. PLoS Med. 4:e297.

Watt RG (2002). Emerging theories into the social determinants of health: implications for oral health promotion. Community Dent Oral Epidemiol 30:241-247.

Weintraub JA, Prakash P, Shain SG, Laccabue M, Gansky SA (2010). Mothers' caries increases odds of children's caries. J Dent Res 89:954-958

Zhan L, Tan S, Den Besten P, Featherstone JD, Hoover CI (2012). Factors related to maternal transmission of mutans streptococci in high-risk children-pilot study. Pediatr Dent 34:e86-e91. 